\title{
Teaching Astronomy at Secondary School Level in Europe
}

\author{
By R. M. Ros \\ Universitat Politècnica de Catalunya, Barcelona, Spain.
}

\section{Introduction}

The status of teaching Astronomy in European countries is variable. Sometimes Astronomy appears as a compulsory subject or as an optional subject, but on many occasions Astronomy appears within another subject, depending on the country. It is even possible for Astronomy not to appear anywhere in the curriculum. But of course the position here is better than in other less developed places. In Europe there are various topics which can be organized into two main groups: aspects related to relative motions and aspects related to properties of light. Some examples of teaching activities and materials in various countries will be described.

It is also necessary to emphasize several initiatives such as the review of Astronomy curricula, the publication of general books on Astronomy for secondary schools and the organisation of new journals to promote Astronomy in schools.

It is essential to mention the new European Association for Astronomy Education (EAAE) founded last November in Athens. This meeting was attended by 100 teachers and astronomy professionals from 17 European countries. It is hoped that this, in conjuntion with the other initiatives, will do much to encourage the study of Astronomy.

\section{Relative Motions.}

In this field, as in others, there is some very interesting material promoted by the Comitè de Liaison Enseignants et Astronomes (CLEA) in France. Denise Wacheux has produced a special umbrella which is used to study the movement of the Sun and celestial sphere in relation to the horizon, and which has very interesting didactic applications in secondary schools. It is possible to change the latitude and to move the umbrella around its axis. Designed for a similar purpose, Roland Szostak (University of Münster, Germany) has interesting material to study the Sun's movement through a half-sphere of plastic on a horizon (Fig. 1). Students use this to plot the sun's trajectory by putting stickers on the half-sphere at regular intervals of time.

There is also a model made using photography from the Permanent Astronomy Seminar of Catalonia (PASC) (Polytechnical University of Catalonia, Spain) made in collaboration with Nicoletta Lanciano (La Sapienza University, Rome) who has wide experience in this field in primary schools. To do this activity the students have to take a collection of photographs of their horizon at school (Fig. 2). With this material they make a cylinder which is supported on a piece of wood. In this oriented model according to the meridian line, they have to put several astronomical concepts such as the local meridian, the equator, the Earth's rotation axis, etc. This model is very important in order to have a vision of the celestial sphere from outside and inside. When the teacher draws the celestial sphere on the blackboard the students have a view from outside. But when the students do a practical activity of course, they are inside the sphere. This kind of change is clarified with this model.

Some simulations of several astronomical aspects have also been developed. Some planetaria (Vittorio Mascellani, Italy) organize activities in which students participate 


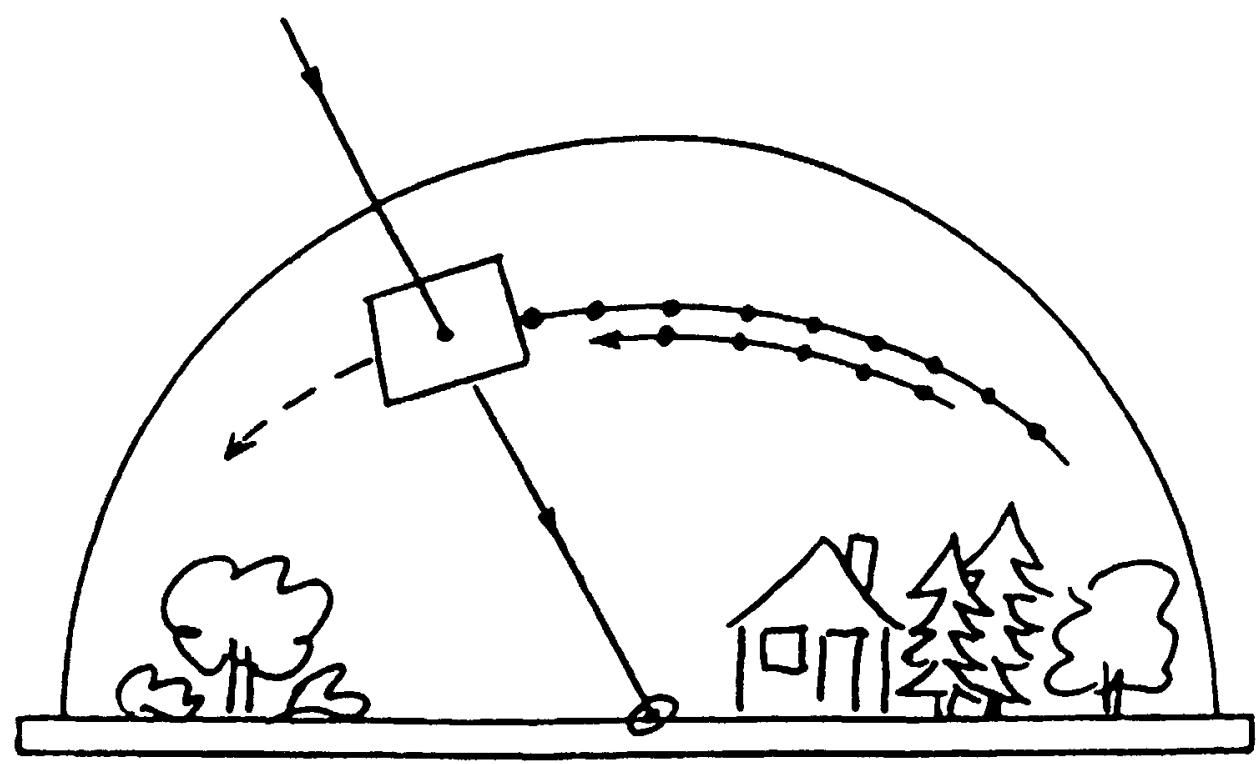

FIGURE 1. The plastic sphere for measuring the path of the Sun above the horizon. (Germany).

to study solar phenomena or to construct a small model of the solar system in the garden. In this type of simulation, members of the Permanent Astronomy Seminars of Euskadi and Catalonia, (PASE and PASC), prepared a model to observe the different kinds of conics on a horizontal plane obtained from the Sun's diurnal movement. This material enable us to explain why the end of the gnomon's shadow describes a conical line. The Sun's ray is simulated by a thread which starts from the Sun's position (in the celestial sphere), passes the end of gnomon and crosses the horizontal plane at the same point as the end of the gnomon's shadow on the horizontal plane. We repeat this simulation at various solar positions. If we observe all the intersection points, we obtain the conical line (ellipses, hyperbolae, parabolae or straight lines). The type of conical line depends on the latitude. The students can only observe one of these conical lines in their city. A similar model was made with small lights which it is possible to switch on and off, one by one, and to observe the shadows on the horizontal plane. This last model has the advantage that it is possible to change the latitude and obtain all the different conical lines in the same model. With this model, the teacher and the students can make an imaginary journey to another country with a different latitude. So they are then able to observe the conical line according to this new latitude.

Still on simulation but this time using computers, there is the work done by Francis Berthomieu (CLEA, France) concerning interactive programs of the Moon's phases and the planets' movements. 


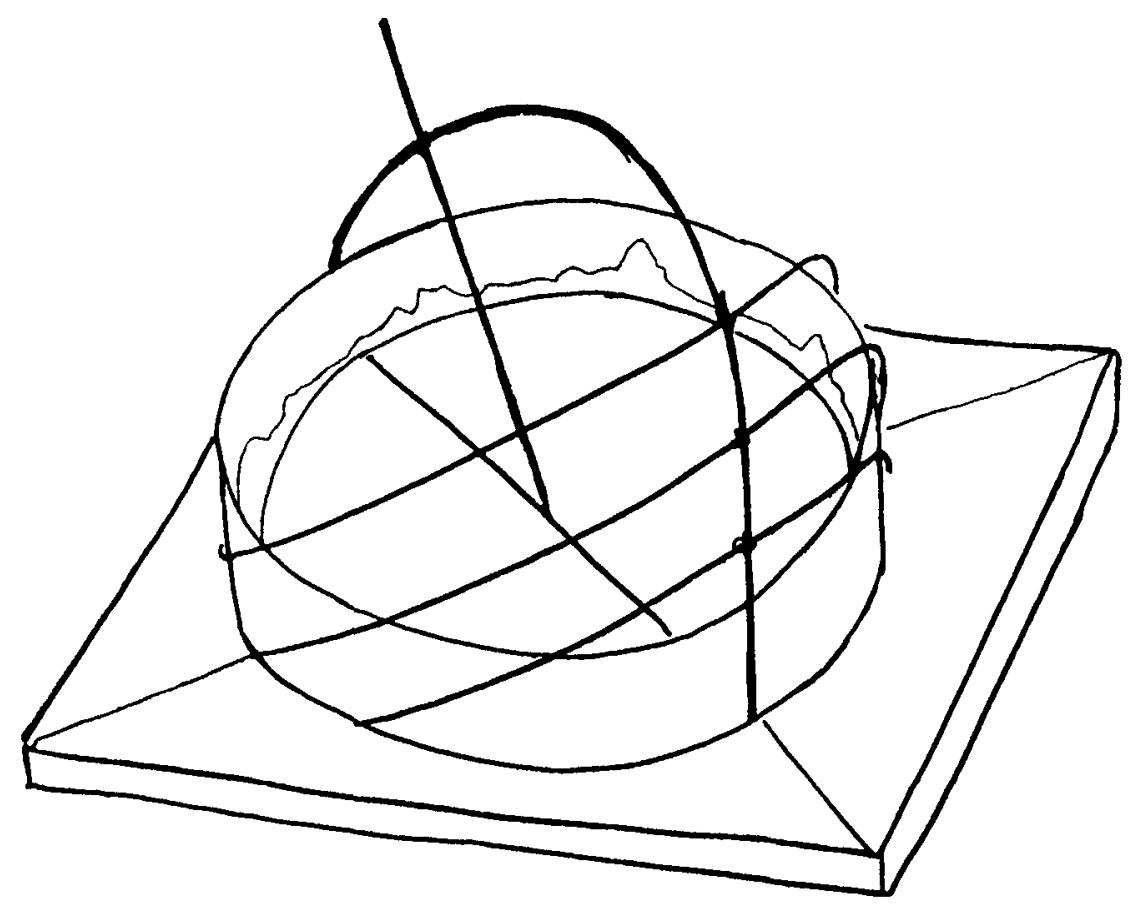

FIGURE 2. Model for studying the specific school's horizon. (Italy and Spain).

\section{Properties of Light}

In the field of Astrophysics and the properties of light, there are interesting experiments developed by Laura Abati (Italy), for example, one to determine the Sun's temperature and another related to Hubble's law. Also a group of activities were developed by Lucienne Gouguenheim (CLEA, France) about spectra and the construction of a spectroscope to observe the spectrum lines. In the same area, Lucette Bottinelli presented a collection of practical experiments which use photocopies of a spectrum to determine its characteristics. She covers very easy cases, for example assigning the spectral type in the Harvard classification (Fig. 3), up to others which are more difficult and elaborate.

The PASE also made a spectroscope constructed with a piece of compact disc placed in a match-box. Along these lines, members of PASC produced a general set of practical activities about the H-R diagram which include the construction of a spectrocope which can be used to read the wavelength of several lines. Another group of professors from the same PASC used photographic activities related to the H-R diagram, magnitudes and variable stars.

In particular this last activity was done in collaboration with members of the Permanent Astronomy Seminar of Aragón (PASA, Spain). The activity on the variable star is very easy to run and has interesting results. The original idea is to test the luminosity curve of a regular variable star, knowing the star's period and epherimedes. If it is necessary, the students have to prepare a simple programme to obtain the timetable to make the observations. For example to observe $\beta P$ erse $i$ it is necessary to know when the star will be at its minimum brightness, but if we observe $\delta C e p h e i$ or RRLyrae it is not necessary because these stars do not have such a spectacular change. In every case the 


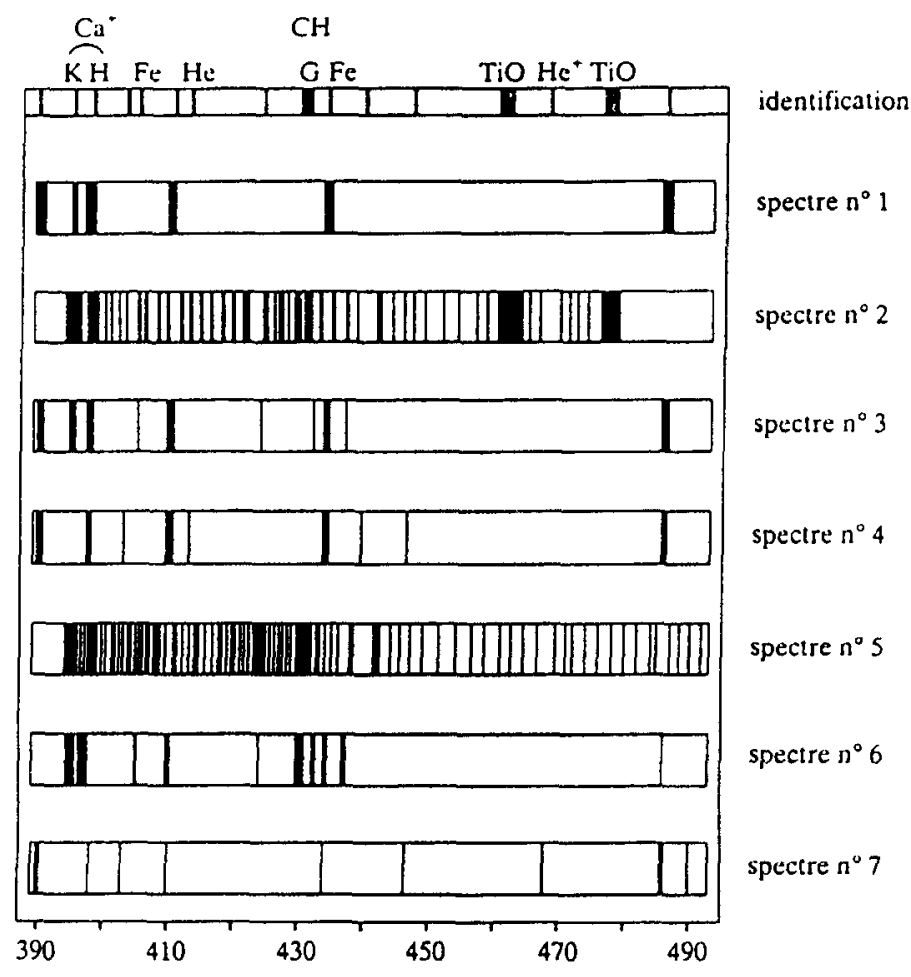

Figure 3. Example of spectral classification. (France).

students have to take some photographs (colour slides) of the star and its constellation, and note the time. When the teacher has some of these photographs, in the classroom the students can calculate the phase and can assign the magnitude for each case, using the method of Argelander. Then, the students obtain some points of the luminosity curve (Fig. 4). Of course it is possible to use the same process to obtain directly the curve without previously knowing it, but this takes longer and does not provide any new information and is boring for students.

The Open University of the United Kingdom offers several projects which can be useful in secondary schools. For example, they have interesting material concerning visual stellar magnitudes and luminosity of the Sun. In particular their study on limiting magnitudes in and around Orion is very appropiate because this constellation is very easy for school pupils to observe when the day is very short.

\section{Aspects of Curricula and Publications.}

In Greece, the Ministry of Education decided to establish a working group, presided over by Panayioti Niarchos and comprising specialists in Astronomy, in order to review the current curriculum for the subject of Astronomy suitable for secondary schools. To encourage and increase the knowledge of Astrophysics among school students, a first summer school in Astronomy this September, will be organized sponsored by the Academy of Athens.

In Portugal, a group of teachers from secondary schools coordinated by Felisbela Martins, is organizing its national association on teaching Astronomy. At present, its first 


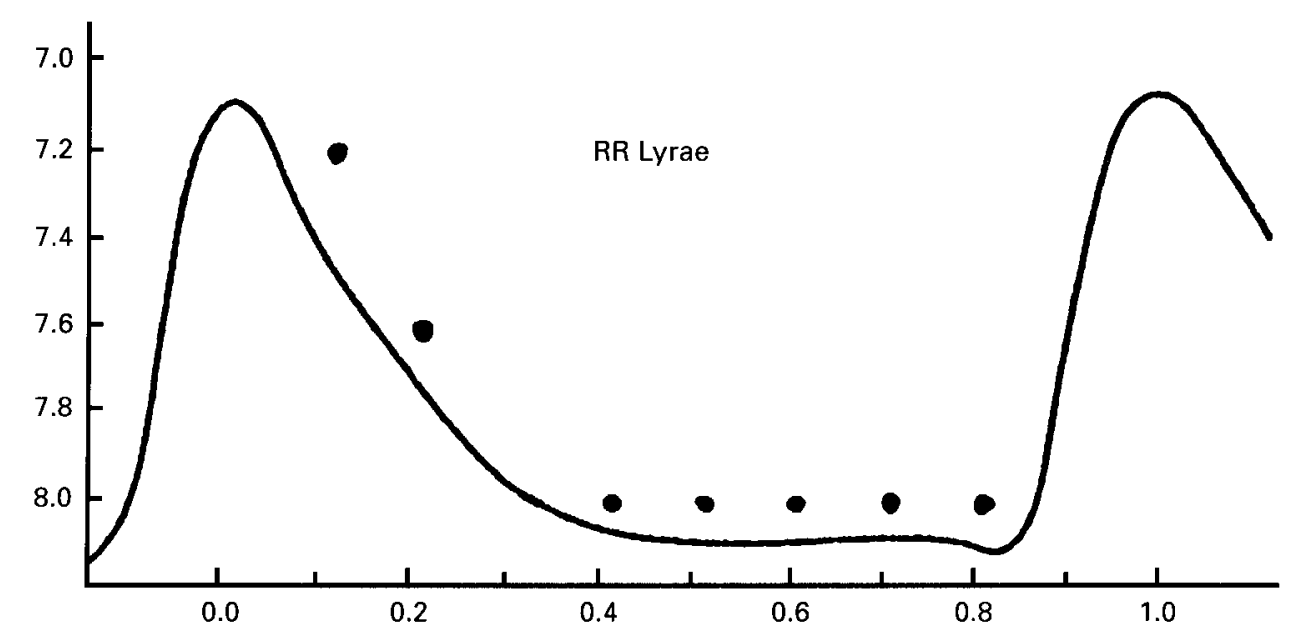

FIGURE 4. Luminosity curve of RR - Lyrae. (Spain).

and principal objective is to organize the 1st National Meeting on Astronomy Education next year.

In some eastern European countries a text book on Astronomy for secondary schools has been recently published. It is necessary for each country to have a book of this kind adapted to its own conditions as it is a vital support in the teaching of the subject. For example in Latvia, Ilgonis Vilks (Riga Observatory, Latvia) finished an Astronomy book for secondary schools and Nikola Nikolov (Sofia University, Bulgaria), Vytautas Straizys (Institute of Theoretical Physics and Astronomy, Lithuania) and E. Kharadze (Tbilisi State University, Georgia) have recently finished other textbooks for the same level.

I do not want to finish my talk without mentioning Edward Kononovich (Moscow University, Russia). He organized an almanac called "The Universe and Ourselves" to cover school problems such as teaching aids, school telescopes and various materials. The main goal of this publication is to help students to understand the place of Mankind in the Universe.

It is necessary to talk about the Blossoms of Science in Israel and their publications and studies on the conceptual frameworks in Astronomy. In particular they observe the secondary school students' progress towards heliocentric concepts, the solar system and astronomical phenomena from a sidereal perspective. Space Science and Astronomy were introduced lately into the curriculum of primary and secondary schools in Israel. Tel Aviv University and the Centre for Educational Technology have produced books and computer software. 


\section{International Contacts.}

To close this paper about teaching Astronomy in Europe it is necessary to speak about the new European Association for Astronomy Education (EAAE), which will improve and promote scientific astronomical education all over Europe in schools at all levels and other institutions involved in teaching Astronomy. The existance of this new EAAE proves the vitality and dynamism of teaching Astronomy in Europe. We can hope this initiative will bear fruit soon.

The interchange with other countries is very enriching. Before finishing I would like to talk a little about the 4th and 5th International Conferences on Teaching Astronomy held in Spain, in 1990 and 1995. Both conferences were very important to promote and stimulate Astronomy in Spain. Teachers and professors from different countries participated in these activities and this promoted several collaborative projects, students exchanges and, of course, a lot of new personal contacts. For example the last meeting, in 1995, gave encouragement to the teachers of secondary schools who were organising the 1st National Meeting of ApEA (Spanish Association for Astronomy Education) held in Caceres last year. It also encouraged us to set up the 1st Summer School of the teaching working group of SEA (Spanish Association of Professional Astronomers) which will be held in Alicante this September.

The work carried out by Kurt Locher from Switzerland can be used as a basis to promote activities between several countries. His studies on Astronomy throughout the history of ancient cultures encourage observational Astronomy today. This common past for a lot of countries is a nexus between them, which it is possible to explore.

Obviously many aspects concerning teaching Astronomy in Europe have not appeared in this brief talk. The intention was not to provide an exhaustive description but simply to give you a taste of what is happening in Europe.

\section{REFERENCES}

Astronomy and the New Technologies, 1995, European Association for Astronomy Education, Eugenides Foundation, Athens.

Proceedings of 5th International Conference on Teaching Astronomy, 1995, Institut de Ciències de l'Educació, Universitat Politècnica de Catalunya, Barcelona. 